\title{
Understanding Central Mechanisms of Acupuncture Analgesia Using Dynamic Quantitative Sensory Testing: A Review
}

\author{
Jiang-Ti Kong, ${ }^{1}$ Rosa N. Schnyer, ${ }^{2}$ Kevin A. Johnson, ${ }^{1}$ and Sean Mackey ${ }^{1}$ \\ ${ }^{1}$ Stanford Systems Neuroscience \& Pain Laboratory, Department of Anesthesiology, Division of Pain Medicine, \\ School of Medicine, Stanford University, 1070 Arastradero Road, Suite 200, Palo Alto, CA 94304, USA \\ ${ }^{2}$ School of Nursing, The University of Texas at Austin, Austin, TX 78701, USA
}

Correspondence should be addressed to Jiang-Ti Kong; calypso1@stanford.edu

Received 24 November 2012; Revised 17 March 2013; Accepted 29 March 2013

Academic Editor: Di Zhang

Copyright (C) 2013 Jiang-Ti Kong et al. This is an open access article distributed under the Creative Commons Attribution License, which permits unrestricted use, distribution, and reproduction in any medium, provided the original work is properly cited.

We discuss the emerging translational tools for the study of acupuncture analgesia with a focus on psychophysical methods. The gap between animal mechanistic studies and human clinical trials of acupuncture analgesia calls for effective translational tools that bridge neurophysiological data with meaningful clinical outcomes. Temporal summation (TS) and conditioned pain modulation $(\mathrm{CPM})$ are two promising tools yet to be widely utilized. These psychophysical measures capture the state of the ascending facilitation and the descending inhibition of nociceptive transmission, respectively. We review the basic concepts and current methodologies underlying these measures in clinical pain research, and illustrate their application to research on acupuncture analgesia. Finally, we highlight the strengths and limitations of these research methods and make recommendations on future directions. The appropriate addition of TS and CPM to our current research armamentarium will facilitate our efforts to elucidate the central analgesic mechanisms of acupuncture in clinical populations.

\section{Overview of Research on Acupuncture Analgesia}

The treatment of chronic pain is the most well-known clinical application of acupuncture in the west $[1,2]$. Acupuncture originated in China more than 2000 years ago and has gained popularity in America since the landmark NIH Consensus Conference in 1997 [3]. Despite broad use, there continues to be ambiguity regarding the efficacy and mechanisms of acupuncture as an analgesic modality. Discrepancies between the results of basic science experiments and clinical trials of acupuncture underscore the controversy surrounding its therapeutic value. The purpose of this review is to outline emerging translational clinical research methods for assessing the central mechanisms of acupuncture analgesia in humans. We begin by summarizing our current understanding of the analgesic mechanisms of acupuncture based on animal and human clinical studies.
1.1. Animal Studies. Animal studies have identified many potential biochemical and neuroanatomical substrates of acupuncture analgesia. Wang et al. [4, 5], Zhao [6], and Han $[7,8]$ have published excellent comprehensive reviews of these studies. From a biochemical standpoint, it appears that acupuncture may alter the metabolism of substrates involved in both the ascending facilitory pathways (N-methyl-Daspartate receptors [9], substance P [10], and interleukin-1 [11]) and the descending inhibitory pain pathways (endogenous opioids [7], serotonin [12], and norepinephrine [13]). From a neuroanatomical standpoint, several central nervous system structures are reported to mediate acupuncture analgesia, including the periaqueductal gray, the nucleus raphe magnus, the locus ceruleus, the arcuate nucleus, the amygdala, and the nucleus accumbens $[4,6]$. It is important to note the link between the biochemical and anatomical substrates. For example, low-frequency electroacupuncture triggers the release of enkephalins and endorphins in the periaqueductal 
gray, the arcuate nucleus, and the caudate nucleus [14]. These structures then send projections to the spinal dorsal horn via the dorsal lateral funiculi [15]. Increases in serotonin release at the nucleus raphe magnus and norepinephrine release in the locus ceruleus are also crucial to analgesia induced by electroacupuncture [13].

In addition to the classic neurotransmitters and anatomical pathways involved in central pain processing, other mechanisms also contribute to acupuncture analgesia [6], including the hypothalamus-pituitary-adrenal axis (regulating peripheral inflammatory response to pain) [16], the autonomic nervous system (regulating local circulation) $[17,18]$, and the glial system [19] (contributing to inflammation around spinal and cerebral neural pathways).

1.2. Human Studies. Although animal studies can provide insight into acupuncture's mechanism of action, establishing the efficacy of acupuncture for treating chronic pain in humans is challenging, owing to the variability of study methods and outcomes [20]. However, an increasing body of robust and rigorous evidence indicates that acupuncture may be an effective intervention for the management of chronic pain [21-23]. Researchers from the Acupuncture Trialists' Collaboration, a group, which was established to synthesize data from high-quality randomized trials on acupuncture for chronic pain, recently published a meta-analysis of 29 clinical trials involving 17,922 patients [23]. The analysis showed that acupuncture consistently yielded greater pain reduction as compared with controls in back and neck pain, arthritis, and headaches. When sham acupuncture was used as the control, the differences were modest but remained statistically significant. Larger differences were seen when standard care (which typically included oral medications and regular physician and physical therapy visits) was used as the control [23].

These results indicate that both specific (i.e., site of needling and stimulation techniques) and nonspecific (i.e., context effects, expectations, etc.) components can contribute to acupuncture's therapeutic effect in treating chronic pain. Understanding the possible mechanisms of these effects in chronic pain remains crucial in elucidating the potential therapeutic value of acupuncture in chronic pain.

1.3. Need for Translational Studies Bridging Mechanisms Observed in Animals to Clinical Populations. Animal studies are of limited benefit in fully modeling the human experience of acupuncture and of chronic pain, and the research methods used in clinical trials provide only a limited understanding of acupuncture's mechanism of action in humans. This gap between animal mechanistic studies and human clinical trials remains one of the greatest challenges in acupuncture research today. The white paper published by the Society of Acupuncture Research (SAR) acknowledged this challenge and proposed goals for future studies [24]. One of the key recommendations was the development of biomarkers that can provide meaningful correlations between physiological effects measured in animal studies and patient-reported outcomes in clinical trials.
To this end, we discuss the emerging translational research methods for assessing the central mechanisms of action of acupuncture analgesia in humans. As background, we first review the basic mechanisms of the central nervous system involved in nociception and human pain perception. Next, we focus the review on two research approaches that likely will emerge as valuable tools for understanding pain processing in acupuncture: temporal summation and conditioned pain modulation. We describe the physiologic mechanism, methodology, and applications of these methods in pain research. Then, we examine the current application of temporal summation and conditioned pain modulation to acupuncture research and make recommendations on future directions.

\section{Nociceptive Pathways and Neural Processing}

2.1. Nociceptive Pathways. Five major components are involved in the perception of pain: (1) the primary peripheral nociceptors; (2) the spinal secondary neurons; (3) the relay neurons (such as those in the thalamus); (4) cortical and subcortical networks responsible for sensory, emotional, and cognitive integration of pain (e.g., the primary sensory cortex, insula, prefrontal cortex, and anterior cingulate cortex); (5) the descending modulatory neurons that originate in subcortical structures (e.g., the periaqueductal grey and locus ceruleus) and project back to the spinal dorsal horn neurons for descending pain processing [25-27].

2.2. Central Nociceptive Processing. Modulation of nociceptive signals occurs beyond the peripheral nociceptors in the central nervous system. This modulation includes processes at the spinal cord and at subcortical and cortical brain structures (components 2, 3, 4, and 5 from above).

First, much of the central nociceptive processing occurs in the spinal dorsal horn $[25,27]$. At least two types of spinal secondary neurons are found in the dorsal horn: the nociceptive-specific (NS) neurons and wide dynamic range (WDR) neurons. The WDR neurons are capable of windup, wherein repetitive noxious stimulation with frequencies above $0.3 \mathrm{~Hz}$ (the natural frequency of the WDR neurons) leads to amplification in output of the WDR neurons [28]. Such increased wind-up is implicated in a variety of chronic pain conditions $[27,29,30]$.

The output of the spinal secondary neurons is dependent on ascending input from the peripheral nociceptors, and it is also modulated by spinal interneurons and descending projections from supraspinal centers. The dynamic balance of these three sources of influence determines the final output from the spinal secondary neurons, which project upward to the relay centers and ultimately to the cerebral cortex for pain perception. This complex interaction of ascending and descending influence on the spinal transmission of pain, commonly referred to as the gate control theory, was originally discovered by Melzack and Wall [31] and has since been validated by many $[32,33]$. 
TABLE 1: Overview of TS and CPM.

\begin{tabular}{|c|c|c|}
\hline & TS & CPM \\
\hline Experimental construct & Repeats of brief noxious stimuli & $\begin{array}{l}\text { A test stimulus measured before and after a } \\
\text { conditioning stimulus }\end{array}$ \\
\hline $\begin{array}{l}\text { Typical magnitudes in healthy } \\
\text { subjects }\end{array}$ & $10-20$ in a $0-100$ visual analog scale (VAS) [42] & $\sim 29 \%$ reduction in pain rating [43] \\
\hline Underlying CNS physiology & $\begin{array}{l}\text { Windup: increased spinal WDR output due to } \\
\text { repetitive C-fiber stimulation at }>0.3 \mathrm{~Hz}\end{array}$ & $\begin{array}{l}\text { DNIC: global reduction of WDR sensitivity due to } \\
\text { a single, heterotopic, noxious stimulation }\end{array}$ \\
\hline $\begin{array}{l}\text { Pain-processing pathways } \\
\text { involved }\end{array}$ & Ascending facilitation of nociceptive input & Descending inhibition of nociceptive input \\
\hline Augmenting factors & $\begin{array}{l}\text { Advanced age [44], female sex }[45,46] \text {, pain } \\
\text { catastrophizing [ } 46-49] \text {, anxiety, fear of pain, } \\
\text { and location (trunk > extremities) [50] }\end{array}$ & \\
\hline Reducing factors & & $\begin{array}{l}\text { Advanced age }[44,51,52] \text {, female sex (mixed } \\
\text { results }[43,45,53]) \text {, pain catastrophizing }[54,55] \text {, } \\
\text { poor sleep }[56,57] \text {, depression }[58] \text {, and opioid } \\
\text { use [59] }\end{array}$ \\
\hline
\end{tabular}

Second, equally important site of central pain processing occurs in the brain via the complex interaction between the cortex and subcortical nuclei [26, 34-36]. The brain is considered crucial for translating nociceptive signals into the conscious perception of pain. Nociceptive signals are relayed from the thalamus to primary and secondary somatosensory regions, and subsequent brain regions are linked to visceral sensation (i.e., insula), emotion (i.e., limbic system), attention (i.e., anterior cingulate), and cognition (i.e., prefrontal cortex). The brain also exerts descending modulation on nociceptive processing via subcortical structures such as the periaqueductal gray (PAG), the rostroventral medulla (RVM), the hypothalamus, the parabrachial nucleus, and the nucleus tractus solitarius. Complex reciprocal interactions exist between the subcortical and cortical centers of pain processing. Eventually, the descending fibers travel in the dorsal lateral funiculus to reach secondary and inter neurons in the spinal dorsal horn [25].

\section{Dynamic Quantitative Sensory Testing}

Quantitative sensory testing (QST), also known as psychophysical testing, refers to tests of sensory perception during the administration of stimuli with predetermined physical properties and following specific protocols [37]. These tests are generally safe and noninvasive for use in human studies, and neuroscience research links these tests to biological underpinnings. Backonja, Arendt-Nielsen, and Pfau [37-39] have published in-depth reviews of quantitative sensory testing.

QST can be subdivided into static QST and dynamic QST $[38,39]$. Static QST typically refers to the measurement of the threshold that primarily reflects states of the peripheral nervous system. Conversely, dynamic QST involves agitation of the pain-perceiving system in a way that exposes a certain mechanism of pain processing beyond the peripheral nervous system. Two extensively studied dynamic paradigms are temporal summation (TS) and conditioned pain modulation (CPM), which represent the ascending facilitatory and descending inhibitory aspects of central pain processing, respectively [38]. Table 1 summarizes the basic concept and characteristics of TS and CPM.

3.1. Temporal Summation. Temporal summation (TS) refers to the increased perception of pain in response to repetitive noxious stimuli delivered at frequencies above $0.3 \mathrm{~Hz}[40,41]$. It is often called "windup pain," or "temporal summation of second pain."

3.1.1. Animal Studies and Molecular Mechanisms. Temporal summation is the behavioral correlate of "windup" of spinal wide dynamic range (WDR) neurons at the dorsal horn $[28,75]$. In animal studies, researchers made single-fiber recordings from the periphery $\mathrm{C}$ fibers and their destined secondary neurons in the spinal dorsal horn. With successive C-fiber activations (by either noxious heat or noxious electrical stimulation) at frequencies over 0.3 pulses per second, WDR neurons displayed increased frequency and amplitude of discharges [75]. These physiologic changes were correlated to behavioral experiments in humans under the same exact stimulation paradigm: they rated the pain with increasing intensity [28]. Thus, TS QST is thought to represent ascending spinal windup of pain processing.

3.1.2. Increase of TS in Chronic Pain and Risk Factors. TS is elevated in a wide variety of pain syndromes, ranging from those that cause idiopathic total body pain (e.g., fibromyalgia [76]) to those considered driven entirely by peripheral factors (e.g., knee arthritis [77]). Increasing evidence suggests that abnormally augmented TS is at least partially responsible for the development of these chronic pain conditions [27]. Furthermore, researchers have identified important risk factors (Table 1) that increase TS, including older age [44], female sex $[45,46]$, psychological factors (anxiety [50], fear [50], and catastrophizing [46-49]), and location of test (the back exhibits higher TS than the upper or lower extremities [50]). 
TABLE 2: Common methods used to generate and compute TS.

\begin{tabular}{|c|c|c|}
\hline Type of stimulus & Experimental paradigms & Variables used to quantify TS \\
\hline Heat pulses & $\begin{array}{l}10-20 \text { heat pulses }(0.5-0.75 \mathrm{~s} \text { each }) \text { delivered at } \\
0.3-0.5 \mathrm{~Hz} \text { either via a continuous contact thermode } \\
\text { [44] or intermittent contact probe [60] }\end{array}$ & $\begin{array}{l}\text { TS magnitude: the difference in pain ratings } \\
\text { between first and last, or first and most painful } \\
\text { pulse, slope of the first few pulses, or the } \\
\text { magnitude of } 5 \text { th pulse }[42,61]\end{array}$ \\
\hline Electrical stimulation & $\begin{array}{l}\text { A single stimulus of a train of five } 1-\mathrm{ms} \text { pulses at } \\
200 \mathrm{~Hz} \text {, repeated } 5 \text { times at } 2 \text { or } 3 \mathrm{~Hz}[62,63]\end{array}$ & $\begin{array}{l}\text { Electrical pain threshold (EP-T): intensity at } \\
\text { which the subject begins to feel pain at the } 4 \text { th or } \\
5 \text { th pulse }[62,63] \text {, or nociceptive withdrawal } \\
\text { reflex threshold (NWR-T), the intensity at which } \\
\text { limb flexion occurs }[64] \text { in response to the } \\
\text { electrical stimulation }\end{array}$ \\
\hline Pin prick & $\begin{array}{l}10 \text { stimuli of } 56 \text { or } 128 \mathrm{mN} \text { are delivered, and pain } \\
\text { ratings for all ten stimuli averaged versus that of a } \\
\text { single stimulus are obtained [65] }\end{array}$ & $\begin{array}{l}\text { Windup ratio: pain of train of } 10 \text { pricks delivered } \\
\text { at } 1 \mathrm{~Hz} \text { over pain of a single prick [65] }\end{array}$ \\
\hline Pressure & $\begin{array}{l}\text { Ten } 1 \text {-s pressure stimuli delivered by an algometer } \\
\text { with } 1 \mathrm{~s} \text { between pulses }[66,67]\end{array}$ & $\begin{array}{l}\text { TS magnitude: difference in pain rating between } \\
\text { the first and 10th stimuli }[66,67]\end{array}$ \\
\hline
\end{tabular}

3.1.3. Methodology for Measurement. Although TS is likely a powerful tool for pain research, the lack of a single, standardized, broadly accepted protocol remains a challenge when interpreting previous work and planning future studies. A variety of noxious stimuli can be used to generate TS, including heat, pin pricks, and electrical stimulation [78]. Although there is no consensus on the quantification of TS [79], 5-20 brief repetitions of identical noxious stimuli are typically given, and the research participant is often asked to rate the changing pain sensation after one or several of the stimuli. Table 2 outlines examples of several commonly used experimental protocols to generate and compute TS.

For heat paradigms, the difference in the pain score between the first and most painful pulse, the slope of pain increase, or even the raw pain score from the fifth pulse can be used to calculate the magnitude of TS $[42,61,79]$. When pin pricks are used as the noxious stimuli, the German Research Network on Neuropathic Pain [65] recommends a standard protocol where either 128 or $56 \mathrm{mN}$ pin tips are applied as a single stimulus and as a series of 10 stimuli given at $1 \mathrm{~Hz}$. The participant is asked to give a single pain rating for the single stimulus and then an average rating for the 10 stimuli repeated at $1 \mathrm{~Hz}$. The ratio of average pain rating of the 10 consecutive stimuli to the rating of the single stimulus is defined as TS or, alternatively, as the windup ratio.

3.1.4. Temporal Summation in Acupuncture Studies. The application of TS to acupuncture research in humans is limited despite the fact that the results of several animal studies indicate that acupuncture produces strong, central modulatory effects and that TS reflects the state of central pain facilitation. Currently, only two clinical studies have been performed involving TS in acupuncture.

In the first study, Zheng et al. [63] randomized 36 healthy volunteers to blindly receiving 25 minutes of electroacupuncture, manual acupuncture, and nonpenetrating sham acupuncture in one leg. The TS threshold for trains of electrical stimulation was assessed before, immediately after, and 24 hours after the treatments on the ipsilateral leg, contralateral leg, and contralateral arm. The results demonstrated that electroacupuncture increased the TS threshold (i.e., reduced TS) in the ipsilateral and contralateral leg up to 24 hours after the treatment. In contrast, manual acupuncture produced no significant change in the TS threshold, although it showed a trend of increase as compared with sham acupuncture. The increase in TS threshold was the greatest in the ipsilateral leg, followed by the contralateral leg; the least change was seen in the contralateral arm, suggesting segmental specificity of the acupuncture interventions. This is one of the very few studies demonstrating that different forms of needle manipulation produced differences in human pain perception linked to a mechanism of central pain processing. Finally, this study demonstrated peripheral influences of acupuncture, as electroacupuncture augmented not only the TS threshold but also the pain detection threshold to singlepulse electrical stimulation.

In the second study, by Tobbackx et al. [80], 39 patients with chronic neck pain due to whiplash injury underwent one session of manual acupuncture (20 minutes) and one session of relaxation therapy (length not specified) in random order with a 1-week between-session washout period. The primary outcomes assessed were pressure pain sensitivity to an analogue algometer and TS scores to 10 consecutive applications of pressure stimuli using the same algometer at a pressure above the pain threshold. The study found that acupuncture caused a greater reduction of the pressure pain threshold as compared with relaxation therapy but produced no change in the TS scores. The authors concluded that in patients with chronic pain, acupuncture does not affect central pain processing.

The inconsistency in the methods used as well as the limited results from these two studies underscore the need for future studies to help further elucidate the role of acupuncture in central pain processing in human subjects. Specifically, both Zheng and Tobbackx demonstrated that after a single session, manual acupuncture did not result in a significant change in TS. However, Zheng was able to demonstrate a significant decrease in TS after electroacupuncture (Tobbackx only studied manual acupuncture). These results suggest that 
electroacupuncture may exert a stronger influence on TS than manual acupuncture. Future studies should be conducted to compare electroacupuncture with manual acupuncture in larger cohorts of patients with chronic pain.

It is also important to note that these studies involved only one acupuncture session. In acupuncture practice, a single session is rarely considered sufficient to produce clinically significant effects for the treatment of chronic pain. Therefore, when translating the results of studies of animal models and healthy human subjects to the clinical pain population, multiple acupuncture sessions with treatment frequency of at least once a week should be considered.

We also recommend performing quantitative sensory testing at multiple anatomical sites adjacent to and at a distance from the site treated for pain. Zheng et al. demonstrated a stronger effect of acupuncture in homotopic versus heterotopic sites, while Tobbackx collected data only on the arm, distal to the neck where the pain and the majority of the needling were located.

Last but not least, there is a need to distinguish the peripheral and central components of acupuncture analgesia. Specifically, Zheng demonstrated increase in the threshold to both temporal summation and to single-pulse protocols, suggesting acupuncture's involvement in both central and peripheral nociceptive modulation. The authors further suggest that a mechanism independent of NMDA blockade, such as peripheral opioid receptor activation [81], may play a role. To test this hypothesis, selective blockade of NMDA and $\mu$-opioid receptors should be used. Furthermore, additional biomarkers of central (e.g., secondary hyperalgesia to capsaicin [82]) and peripheral pain processing (pressure and heat pain threshold [38]) may also be used to profile the pain modulatory mechanisms of acupuncture.

3.2. Conditioned Pain Modulation. Conditioned pain modulation (CPM) refers to the phenomenon whereby a noxious stimulus at one body part results in reduced pain perception to another noxious stimulus at a distant, heterotopic body part $[83,84]$. The first stimulus is referred to as the conditioning stimulus; the second stimulus, whose pain rating decreases after the application of the conditioning stimulus, is referred to as the test stimulus [84]. CPM has been shown to be a separate phenomenon from cognitive distraction [85, 86]. A variety of other terms have also been used to describe CPM, such as "pain inhibiting pain," "heterotopic noxious conditioning stimulation," and "counterirritation $[83,84]$." CPM was also referred to as diffuse noxious inhibitory control (DNIC). However, international experts have agreed to distinguish DNIC, a neurophysiologic process, from CPM, a behavioral correlate of this process (see below) [84].

3.2.1. Animal Studies and Molecular Mechanisms. CPM is the behavioral correlate to diffuse noxious inhibitory control (DNIC) [84], an inhibitory mechanism involving the spinalbulbo-spinal loop in animal neurophysiological studies [87]. In 1979, Le Bars et al. discovered that when noxious stimuli (A- $\delta$ - or C-fiber-mediated) are applied anywhere outside the excitatory receptive field of a spinal or trigeminal
WDR neuron, the response to any noxious input within its receptive field is profoundly inhibited [88, 89]. Le Bars' group subsequently found that DNIC is mediated by the subnucleus reticularis dorsalis (SRD) in the caudal medulla [90], which receives noxious input via pathways in the ventral lateral quadrant of the spinal cord [91], and sends global inhibitory signals to WDR neurons from all spinal levels via the dorsolateral funiculi [92]. Finally, the strong correlation between the signal reduction in the WDR neurons and the pain reduction in a CPM paradigm, in both extent and time course, supports the notion that CPM is the behavioral correlate of DNIC [93-95].

3.2.2. Decrease of CPM in Chronic Pain and Risk Factors. Like TS, CPM is altered in many chronic pain conditions, such as fibromyalgia [96], tension-type headache [97], irritable bowel syndrome [98], and arthritis of the hip [99]. Rather than an increase as with TS, a decrease in CPM is seen. As shown in Table 1, the risk factors for decreased CPM are similar to those for increased TS. These include older age [44, 51, 52], female sex $[43,45,53]$, and psychological factors such as catastrophizing $[54,55]$. However, the relationship between female sex and decreased CPM is less straight forward as some studies showed clear increase in CPM associated with female sex, while others did not find such association $[43,45$, 53]. Large variations in methodology may partially contribute to this discrepancy $[43,53]$. Furthermore, chronic opioid use [59], depressed mood [58], and poor sleep also decrease CPM $[56,57]$.

3.2.3. Measurement Methodology. There is no single, standard protocol for measuring CPM. Table 3 summarizes the key components in generating CPM and demonstrates examples of their variability. Pud et al. [43] published an excellent review of CPM methods. In short, a test stimulus is measured before the application of the conditioning stimulus to obtain a baseline and is measured again during or after the application of the conditioning stimulus to quantify the magnitude of CPM relative to the baseline. The noxious test and conditioning stimuli are typically administered at anatomically distinct locations.

The most common conditioning stimulus is a cold water bath applied to the contralateral hand. However, other conditioning stimuli have been used, including isotonic saline injections and heat pain. It is the general consensus that the conditioning stimulus must be noxious in order to trigger CPM $[43,53]$. Once the noxious threshold has been exceeded, the intensity of the conditioning stimulus may not matter, according to reports by Granot et al. [68] and Nir et al. [100]. The duration of the conditioning stimulus is usually between 30 seconds and 2 minutes for the cold water bath [53].

In contrast to the conditioning stimulus, there is a large variation in the choice of test stimulus. Pain recordings of a standard stimulus or pain thresholds from any type of stimulation (electrical, chemical, heat, pressure, etc.) can be used [43]. The magnitude of CPM is measured by the change from baseline in the test stimulus to after the conditioning stimulus is applied. The CPM effect appears to peak during 
TABLE 3: Examples of varied parameters in generating CPM.

\begin{tabular}{ll}
\hline Parameter & Examples \\
\hline Conditioning stimulus & $\begin{array}{l}\text { Cold water bath }\left(0-10^{\circ} \mathrm{C}\right)[68], \text { heat (thermode or water bath) [69], hypertonic } \\
\text { saline injection [70], and inflated blood pressure cuff [71] } \\
\text { Pain detection thresholds [69], rating of a predetermined single pain stimulus [68], } \\
\text { and TS protocols [72] } \\
\text { Testing stimulus }\end{array}$ \\
$\begin{array}{l}\text { Varies widely: from during the conditioning stimulus [73] up to 30 min after the } \\
\text { conditioning stimulus [74] }\end{array}$ \\
$\begin{array}{l}\text { Large variation in the relative distance between the testing and conditioning stimuli: } \\
\text { for example, upper body to upper body [68] versus upper body to lower body [51] } \\
\text { Location of stimulus }\end{array}$ \\
$\begin{array}{l}\text { Relative or absolute changes in threshold measures or ratings of predetermined pain } \\
\text { stimulus [43] }\end{array}$ \\
\hline
\end{tabular}

the application of the conditioning stimulus and fades rapidly from 5 to 10 minutes after the conditioning stimulus ceases $[43,53,101,102]$. One report indicates that the approximate median magnitude of CPM represents about a $29 \%$ decrease in pain rating, regardless of the test stimulus used [43]. There is some indication that CPM is stronger when the test and conditioning stimuli are applied at a greater anatomical distance from the CPM stimulus site (e.g., CPM is stronger for the hand-to-contralateral leg than for the handto-contralateral hand) $[43,87]$.

In summary, the best means of capturing robust CPM is to use a strong, noxious conditioning stimulus (such as cold immersion of the contralateral distal extremity) and measure the change in the test stimulus during the latter part of the conditioning stimulus. As with TS, significant variations exist in the methodologies used to generate and compute CPM, making it difficult to make comparisons across studies and subjects. Future efforts should focus on identifying a standardized, broadly accepted protocol for CPM.

3.2.4. CPM in Acupuncture Studies. Similar to TS, the use of CPM to study acupuncture analgesia is limited. There are only two direct studies on acupuncture analgesia and CPM/DNIC, both of which focused on the question of whether acupuncture analgesia is equivalent to CPM/DNIC. To date, no one has studied how acupuncture stimulation may modulate the extent of DNIC.

The first acupuncture-DNIC study was carried out by Bing et al. [103]. Output from WDR neurons in the trigeminal nucleus of rodents was measured using the patch-clamp technique. The conditioning stimuli consisted of manual acupuncture applied to Zusanli (a classic acupuncture point) and to an adjacent nonacupuncture point, both located on the right hind limb, and a standard noxious stimulusimmersion of the left hind limb in a $48^{\circ} \mathrm{C}$ hot water bath. All three stimuli resulted in a similar degree of inhibition in the firing of the trigeminal WDR neurons (72.5\% and 78.5\%). Furthermore, the inhibition resulting from all three stimuli demonstrated a similar time course for decay and a similar response to naloxone, which reversed the inhibition by about $30 \%$. The authors concluded that acupuncture maneuvers trigger the neural mechanisms involved in DNIC.
The second acupuncture-DNIC (CPM) study was done in healthy human volunteers using a crossover design [104]. It directly compared the effects of acupuncture, sham acupuncture, and a $1.5^{\circ} \mathrm{C}$ cold water bath (as a conditioning stimulus in the upper extremity). The test stimulus was the pressure pain threshold at the second toe. The verum acupuncture involved the penetration of Hegu (a classic acupuncture point) with a needle without manipulation, and retaining the needle for 5 minutes. The sham acupuncture involved the tapping and placement of a nonpenetrating Streitberger device [105] at Hegu for 5 minutes. The results showed that the cold bath resulted in much stronger increase in the pressure pain threshold as compared with verum and sham acupuncture. Furthermore, there was no statistically significant difference between the verum and sham. The authors concluded that acupuncture as performed in this trial exerted a small analgesic effect not different from placebo and that the analgesic effect was unlike CPM.

It is difficult to compare these studies because of several reasons. First, the test and conditioning stimuli differed significantly between the two studies. Second, as the authors of the second paper mentioned, their acupuncture needling was only minimally painful (pain score about $2.4 \pm 1.5$ out of a 10-point scale). Because prior studies have shown that CPM will only work when the conditioning stimulus is beyond the noxious threshold, it is not surprising that acupuncture did not trigger CPM in these studies. Third, Deqi, a unique composite of sensations (such as deep ache and tingling sensation) considered essential for clinical efficacy according to traditional Chinese medicine [106], was not elicited in the second study. In real clinical practice, the acupuncturist aims to achieve Deqi, retains the needles in the body for between 15 and 20 minutes, and often uses more than one needle [107]. Therefore, the treatment performed in the second study does not represent typical clinical practice. Future studies should focus on adapting CPM for the clinical study of acupuncture analgesia using acupuncture methods that are consistent with clinical practice.

Last but not least, the duration of CPM/DNIC is shortlived. Directly comparing acupuncture with CPM will not help understand the clinically relevant long-term analgesia by acupuncture. Because DNIC/CPM is considered to play a role in mediating pain perception in chronic pain conditions [27, 108,109 ], it would be more relevant to study how acupuncture 
influences CPM rather than simply to treat acupuncture as a form of transient conditioning stimulus.

\subsection{Current Trends and Future Directions QST in Acupuncture Research}

3.3.1. Clinical Applications of QST. Quantitative sensory testing is increasingly used in clinical research as a helpful marker for central and peripheral nociceptive processing $[38,39,110]$. Specifically, researchers are applying TS and CPM to the following three categories of translational research: (1) to phenotype patient subgroups based on different underlying pain mechanisms $[27,53,109,111]$; (2) to predict response to treatment based on mechanistic phenotypes thus determined [112-116]; and (3) to characterize the central modulatory effects of new therapies [99, 117-121]. For example, as mentioned separately in the TS and CPM sections, many chronic pain conditions display increased TS and/or reduced $\mathrm{CPM}$ as compared with healthy controls. These conditions include fibromyalgia, TMJ disorder, headaches, irritable bowel syndrome, back pain, and arthritis of hip and knee [27]. The next step, then, is to select the appropriate therapies that specifically address an individual patient's mechanistic deficit(s). Recently, Yarnitsky et al. demonstrated this concept of mechanism-based treatment of pain in a landmark study [116]. He found that deficient descending inhibition, as reflected by low CPM, predicted clinical response to duloxetine, an antidepressant that augments descending inhibition by increasing serotonin and norepinephrine levels in the central nervous system [122]. This study represents a future of personalized pain treatment, where QST fingerprinting provides key information on the mechanism of an individual's pain condition. Last but not least, TS and CPM have been used to characterize the central modulatory effects of many drugs and interventions, such as ketamine [123], gabapentin [117], surgery [120], and exercise [121].

3.3.2. Limitations of Current QST Research. Despite the advances described here, there are still significant methodological barriers to the wide utilization of QST in clinical research. First, although the temporal stability of TS and CPM has been established in healthy volunteers $[42,66,124]$, it has not been characterized in chronic pain patients [110]. Without stability data in this population, it is difficult to discern if a change in TS or CPM is due to the therapeutic intervention or to the natural fluctuation in these measures. Second, a variety of methods have been used to generate and compute TS $[42,79]$ and CPM [43], which makes it challenging to make comparisons across studies. Third, individual TS and CPM responses vary widely. For example, a standard thermal TS protocol may not capture TS from $40 \%$ to $60 \%$ of the individuals tested $[61,125]$. This variability imposes restrictions on the external validity of studies involving TS and CPM, particularly in longitudinal studies where some degrees of TS and CPM are expected at baseline. Our group is actively searching for solution to all 3 issues by assessing the stability of TS and CPM in patients with chronic pain, developing universal protocols to capture TS and CPM in most individuals, and investigating the physiologic bases of the between-individual variations in the response to TS and CPM tests.

In summary, while there is a large body of literature on using TS and CPM to study chronic pain, limitations in methodology still exist. Future studies should address the following three issues: (1) the uncertainty in the temporal stability of TS and CPM in chronic pain conditions; (2) the lack of a universal protocol; (3) the large between-individual variability in both TS and CPM.

3.3.3. QST in Acupuncture Research. The literature contains only sparse data on the use of QST in human acupuncture research. This may be due, in part, to lack of awareness. Advances are being made in applying QST to understand the mechanisms of chronic pain $[27,38,53,78,109]$ and medications $[29,112,116-118,126]$, and we believe there is a definitive role for QST in the study of acupuncture analgesia.

We make the following practical recommendations for utilizing QST in clinical acupuncture studies. First, because the stability of TS and CPM has not been established in the clinical pain population, we recommend a parallel group design with one group representing the natural course of the disease (waitlist control) and other groups representing active and sham interventions. Second, although there is no single standardized protocol for TS or CPM, there are both more and less established protocols. We recommend choosing the more established protocols. For example, the OPERA trial of several thousands of patients with oral facial pain used reliable heat and pressure pain paradigms to examine TS and CPM [111]. Another example is the comprehensive set of QST measures that encompass both the dynamic and static measures developed by the German Research Network in Neuropathic Pain (DFNS) [65]. With a strong focus on peripheral pathologies, this protocol has been validated on a variety of neuropathic pain syndromes and has been shown to be consistent [127]. Furthermore, both the OPERA and the DFNS protocols include traditional threshold measures to a variety of sensory stimuli. Unlike CPM and TS, these threshold measures offer additional insights on peripheral nociceptive processes. Last but not least, we recommend a two-step approach to determine whether acupuncture leads to analgesia by modifying the central pain-processing pathways. First, research should test whether acupuncture will lead to changes in dynamic QST parameters such as TS and CPM. Second, studies should investigate whether such changes lead to improvement of pain and function.

\section{Conclusions}

Numerous animal studies suggest that acupuncture leads to analgesia via powerful central pain modulatory mechanisms. However, little is known about whether and how these findings may translate to clinically meaningful outcomes. TS and CPM are emerging behavioral correlates of ascending excitatory and descending inhibitory limbs of central pain 
modulation. Both TS and CPM have been widely used in clinical pain research, yet their application to the understanding of acupuncture analgesia is limited. We encourage greater use of TS and CPM in acupuncture research, in conjunction with other psychophysical tools such as pain detection thresholds, and with appropriate attention given to the limitations of these psychophysical methods. The appropriate adaption of dynamic (TS and CPM) and static QST measures (pain detection threshold) will help advance our understanding of the true mechanisms of acupuncture analgesia in human clinical populations.

\section{Abbreviations}

AA: Acupuncture analgesia

TS: Temporal summation

CPM: Conditioned pain modulation

QST: Quantitative sensory testing

DNIC: Diffuse noxious inhibitory control

NS: $\quad$ Nociceptive specific

WDR: Wide dynamic range

PAG: Periaqueductal gray

RVM: Rostroventral medulla

SDR: Subnucleus dorsalis reticularis

S1: $\quad$ Somatosensory area 1

S2: $\quad$ Somatosensory area 2.

\section{Acknowledgments}

The authors thank Jarred Younger, $\mathrm{PhD}$, and Katie Martucci, $\mathrm{PhD}$, for constructive feedback on the content and Maureen Donohue, BA, for critical assistance in the preparation of this paper.

\section{References}

[1] A. Burke, D. M. Upchurch, C. Dye, and L. Chyu, "Acupuncture use in the United States: findings from the national health interview survey," Journal of Alternative and Complementary Medicine, vol. 12, no. 7, pp. 639-648, 2006.

[2] A. Vincent, K. M. Kruk, S. S. Cha, B. A. Bauer, and D. P. Martin, "Utilisation of acupuncture at an academic medical centre," Acupuncture in Medicine, vol. 28, no. 4, pp. 189-190, 2010.

[3] Y. Zhang, L. Lao, H. Chen, and R. Ceballos, "Acupuncture use among american adults: what acupuncture practitioners can learn from national health interview survey 2007?" EvidenceBased Complementary and Alternative Medicine, vol. 2012, Article ID 710750, 8 pages, 2012.

[4] S. M. Wang, Z. N. Kain, and P. White, "Acupuncture analgesia: I. The scientific basis," Anesthesia and Analgesia, vol. 106, no. 2, pp. 602-610, 2008.

[5] S. M. Wang, Z. N. Kain, and P. F. White, "Acupuncture analgesia: II. Clinical considerations," Anesthesia and Analgesia, vol. 106, no. 2, pp. 611-621, 2008.

[6] Z. Q. Zhao, "Neural mechanism underlying acupuncture analgesia," Progress in Neurobiology, vol. 85, no. 4, pp. 355-375, 2008.

[7] J. S. Han, "Acupuncture and endorphins," Neuroscience Letters, vol. 361, no. 1-3, pp. 258-261, 2004.
[8] J. S. Han, "Acupuncture analgesia: areas of consensus and controversy," Pain, vol. 152, no. 3, pp. S41-S48, 2011.

[9] R. X. Zhang, L. Wang, X. Wang, K. Ren, B. M. Berman, and L. Lao, "Electroacupuncture combined with MK-801 prolongs anti-hyperalgesia in rats with peripheral inflammation," Pharmacology Biochemistry and Behavior, vol. 81, no. 1, pp. 146-151, 2005.

[10] N. Yonehara, T. Sawada, H. Matsuura, and R. Inoki, "Influence of electro-acupuncture on the release of substance $\mathrm{P}$ and the potential evoked by tooth pulp stimulation in the trigeminal nucleus caudalis of the rabbit," Neuroscience Letters, vol. 142, no. 1, pp. 53-56, 1992.

[11] R. X. Zhang, A. Li, B. Liu et al., "Electroacupuncture attenuates bone cancer pain and inhibits spinal interleukin- $1 \beta$ expression in a rat model," Anesthesia and Analgesia, vol. 105, no. 5, pp. 1482-1488, 2007.

[12] W. B. Hu, Z. J. Wu, and K. M. Wang, "Progress of researches on involvement of serotonin in the central nervous system in acupuncture analgesia and other effects," Zhen Ci Yan Jiu, vol. 37, no. 3, pp. 247-251, 2012.

[13] Y. Zhang, R. X. Zhang, M. Zhang et al., "Electroacupuncture inhibition of hyperalgesia in an inflammatory pain rat model: involvement of distinct spinal serotonin and norepinephrine receptor subtypes," The British Journal of Anaesthesia, vol. 109, no. 2, pp. 245-252, 2012.

[14] L. F. He, "Involvement of endogenous opioid peptides in acupuncture analgesia," Pain, vol. 31, no. 1, pp. 99-121, 1987.

[15] A. Li, Y. Wang, J. Xin et al., "Electroacupuncture suppresses hyperalgesia and spinal Fos expression by activating the descending inhibitory system," Brain Research, vol. 1186, no. 1, pp. 171-179, 2007.

[16] R. X. Zhang, L. Lao, X. Wang et al., "Electroacupuncture attenuates inflammation in a rat model," Journal of Alternative and Complementary Medicine, vol. 11, no. 1, pp. 135-142, 2005.

[17] K. Kubo, H. Yajima, M. Takayama, T. Ikebukuro, H. Mizoguchi, and N. Takakura, "Changes in blood circulation of the contralateral Achilles tendon during and after acupuncture and heating," International Journal of Sports Medicine, vol. 32, no. 10, pp. 807813, 2011.

[18] C. S. Huang and Y. F. Tsai, "Somatosympathetic reflex and acupuncture-related analgesia," Chinese Journal of Physiology, vol. 52, no. 5, pp. 345-357, 2009.

[19] J. M. Kang, H. J. Park, Y. G. Choi et al., "Acupuncture inhibits microglial activation and inflammatory events in the MPTPinduced mouse model," Brain Research, vol. 1131, no. 1, pp. 211219, 2007.

[20] E. Ernst, M. S. Lee, and T. Y. Choi, "Acupuncture: does it alleviate pain and are there serious risks? A review of reviews," Pain, vol. 152, no. 4, pp. 755-764, 2011.

[21] B. M. Berman, H. H. Langevin, C. M. Witt, and R. Dubner, "Acupuncture for chronic low back pain," The New England Journal of Medicine, vol. 363, no. 5, pp. 454-461, 2010.

[22] A. Hopton and H. MacPherson, "Acupuncture for chronic pain: is acupuncture more than an effective placebo? A systematic review of pooled data from meta-analyses," Pain Practice, vol. 10, no. 2, pp. 94-102, 2010.

[23] A. J. Vickers, A. M. Cronin, A. C. Maschino et al., "Acupuncture for chronic pain: individual patient data meta-analysis," Archives of Internal Medicine, vol. 172, no. 19, pp. 1444-1453, 2012. 
[24] H. M. Langevin et al., "Paradoxes in acupuncture research: strategies for moving forward," Evidence-Based Complementary and Alternative Medicine, vol. 2011, Article ID 180805, 11 pages, 2011.

[25] R. D'Mello and A. H. Dickenson, "Spinal cord mechanisms of pain," The British Journal of Anaesthesia, vol. 101, no. 1, pp. 8-16, 2008.

[26] M. J. Millan, "Descending control of pain," Progress in Neurobiology, vol. 66, no. 6, pp. 355-474, 2002.

[27] K. Phillips and D. J. Clauw, "Central pain mechanisms in chronic pain states-maybe it is all in their head," Best Practice \& Research Clinical Rheumatology, vol. 25, no. 2, pp. 141-154, 2011.

[28] D. D. Price, "Characteristics of second pain and flexion reflexes indicative of prolonged central summation," Experimental Neurology, vol. 37, no. 2, pp. 371-387, 1972.

[29] P. K. Eide, "Wind-up and the NMDA receptor complex from a clinical perspective," The European Journal of Pain, vol. 4, no. 1, pp. $5-15,2000$.

[30] R. Kuner, "Central mechanisms of pathological pain," Nature Medicine, vol. 16, no. 11, pp. 1258-1266, 2010.

[31] R. Melzack and P. D. Wall, "Pain mechanisms: a new theory," Science, vol. 150, no. 3699, pp. 971-979, 1965.

[32] A. H. Dickenson, "Gate control theory of pain stands the test of time," The British Journal of Anaesthesia, vol. 88, no. 6, pp. 755-757, 2002.

[33] M. Moayedi and K. D. Davis, "Theories of pain: from specificity to gate control," Journal of Neurophysiology, vol. 109, no. 1, pp. 5-12, 2012.

[34] A. V. Apkarian, M. C. Bushnell, R. D. Treede, and J. K. Zubieta, "Human brain mechanisms of pain perception and regulation in health and disease," The European Journal of Pain, vol. 9, no. 4, pp. 463-484, 2005.

[35] A. D. Craig, "Pain mechanisms: labeled lines versus convergence in central processing," Annual Review of Neuroscience, vol. 26, pp. 1-30, 2003.

[36] S. C. Mackey and F. Maeda, "Functional imaging and the neural systems of chronic pain," Neurosurgery Clinics of North America, vol. 15, no. 3, pp. 269-288, 2004.

[37] M. M. Backonja, D. Walk, R. R. Edwards et al., "Quantitative sensory testing in measurement of neuropathic pain phenomena and other sensory abnormalities," The Clinical journal of pain, vol. 25, no. 7, pp. 641-647, 2009.

[38] L. Arendt-Nielsen and D. Yarnitsky, "Experimental and clinical applications of quantitative sensory testing applied to skin, muscles and viscera," Journal of Pain, vol. 10, no. 6, pp. 556-572, 2009.

[39] D. B. Pfau, C. Geber, F. Birklein, and R. D. Treede, "Quantitative sensory testing of neuropathic pain patients: potential mechanistic and therapeutic implications," Current Pain and Headache Reports, vol. 16, no. 3, pp. 199-206, 2012.

[40] I. H. Wagman and D. D. Price, "Responses of dorsal horn cells of M. mulatta to cutaneous and sural nerve A and C fiber stimuli," Journal of Neurophysiology, vol. 32, no. 6, pp. 803-817, 1969.

[41] L. M. Mendell, "Physiological properties of unmyelinated fiber projection to the spinal cord," Experimental Neurology, vol. 16, no. 3, pp. 316-332, 1966.

[42] J. T. Kong, K. A. Johnson, R. R. Balise, and S. Mackey, “Testretest reliability of thermal temporal summation using an individualized protocol," Journal of Pain, vol. 14, no. 1, pp. 79-88, 2013.
[43] D. Pud, Y. Granovsky, and D. Yarnitsky, "The methodology of experimentally induced diffuse noxious inhibitory control (DNIC)-like effect in humans," Pain, vol. 144, no. 1-2, pp. 16-19, 2009.

[44] R. R. Edwards and R. B. Fillingim, "Effects of age on temporal summation and habituation of thermal pain: clinical relevance in healthy older and younger adults," Journal of Pain, vol. 2, no. 6, pp. 307-317, 2001.

[45] R. B. Fillingim, C. D. King, M. C. Ribeiro-Dasilva, B. RahimWilliams, and J. L. Riley, "Sex, gender, and pain: a review of recent clinical and experimental findings," Journal of Pain, vol. 10, no. 5, pp. 447-485, 2009.

[46] S. Z. George, V. T. Wittmer, R. B. Fillingim, and M. E. Robinson, "Sex and pain-related psychological variables are associated with thermal pain sensitivity for patients with chronic low back pain," Journal of Pain, vol. 8, no. 1, pp. 2-10, 2007.

[47] R. R. Edwards, M. T. Smith, G. Stonerock, and J. A. Haythornthwaite, "Pain-related catastrophizing in healthy women is associated with greater temporal summation of and reduced habituation to thermal pain," Clinical Journal of Pain, vol. 22, no. 8, pp. 730-737, 2006.

[48] B. R. Goodin, T. L. Glover, A. Sotolongo et al., “The association of greater dispositional optimism with less endogenous pain facilitation is indirectly transmitted through lower levels of pain catastrophizing," Journal of Pain, vol. 14, no. 2, pp. 126-135, 2013.

[49] J. L. Rhudy, S. L. Martin, E. L. Terry et al., "Pain catastrophizing is related to temporal summation of pain but not temporal summation of the nociceptive flexion reflex," Pain, vol. 152, no. 4, pp. 794-801, 2011.

[50] M. E. Robinson, J. E. Bialosky, M. D. Bishop, D. D. Price, and S. Z. George, "Supra-threshold scaling, temporal summation, and after-sensation: relationships to each other and anxiety/fear," Journal of Pain Research, vol. 3, pp. 25-32, 2010.

[51] M. Larivière, P. Goffaux, S. Marchand, and N. Julien, "Changes in pain perception and descending inhibitory controls start at middle age in healthy adults," Clinical Journal of Pain, vol. 23, no. 6, pp. 506-510, 2007.

[52] L. L. Washington, S. J. Gibson, and R. D. Helme, "Age-related differences in the endogenous analgesic response to repeated cold water immersion in human volunteers," Pain, vol. 89, no. 1, pp. 89-96, 2000.

[53] G. van Wijk and D. S. Veldhuijzen, "Perspective on diffuse noxious inhibitory controls as a model of endogenous pain modulation in clinical pain syndromes," Journal of Pain, vol. 11, no. 5, pp. 408-419, 2010.

[54] B. R. Goodin, L. McGuire, M. Allshouse et al., "Associations between catastrophizing and endogenous pain-inhibitory processes: sex differences," Journal of Pain, vol. 10, no. 2, pp. 180190, 2009.

[55] I. Weissman-Fogel, E. Sprecher, and D. Pud, "Effects of catastrophizing on pain perception and pain modulation," Experimental Brain Research, vol. 186, no. 1, pp. 79-85, 2008.

[56] R. R. Edwards, E. Grace, S. Peterson, B. Klick, J. A. Haythornthwaite, and M. T. Smith, "Sleep continuity and architecture: associations with pain-inhibitory processes in patients with temporomandibular joint disorder," The European Journal of Pain, vol. 13, no. 10, pp. 1043-1047, 2009.

[57] M. T. Smith, R. R. Edwards, U. D. McCann, and J. A. Haythomthwaite, "The effects of sleep deprivation on pain inhibition and spontaneous pain in women," Sleep, vol. 30, no. 4, pp. 494-505, 2007. 
[58] J. B. De Souza, S. Potvin, P. Goffaux, J. Charest, and S. Marchand, "The deficit of pain inhibition in fibromyalgia is more pronounced in patients with comorbid depressive symptoms," Clinical Journal of Pain, vol. 25, no. 2, pp. 123-127, 2009.

[59] K. C. Ram, E. Eisenberg, M. Haddad, and D. Pud, “Oral opioid use alters DNIC but not cold pain perception in patients with chronic pain-new perspective of opioid-induced hyperalgesia," Pain, vol. 139, no. 2, pp. 431-438, 2008.

[60] K. G. Raphael, "Temporal summation of heat pain in temporomandibular disorder patients," Journal of orofacial pain, vol. 23, no. 1, pp. 54-64, 2009.

[61] R. J. Anderson, J. G. Craggs, J. E. Bialosky et al., “Temporal summation of second pain: variability in responses to a fixed protocol," The European Journal of Pain, vol. 17, no. 1, pp. 67-74, 2013.

[62] L. Arendt-Nielsen, J. Brennum, S. Sindrup, and P. Bak, "Electrophysiological and psychophysical quantification of temporal summation in the human nociceptive system," European Journal of Applied Physiology and Occupational Physiology, vol. 68, no. 3, pp. 266-273, 1994.

[63] Z. Zheng, S. J. Q. Feng, C. D. Costa, C. G. Li, D. Lu, and C. C. Xue, "Acupuncture analgesia for temporal summation of experimental pain: a randomised controlled study," The European Journal of Pain, vol. 14, no. 7, pp. 725-731, 2010.

[64] J. A. B. Manresa, M. B. Jensen, and O. K. Andersen, "Introducing the reflex probability maps in the quantification of nociceptive withdrawal reflex receptive fields in humans," Journal of Electromyography and Kinesiology, vol. 21, no. 1, pp. 67-76, 2011.

[65] R. Rolke, R. Baron, C. Maier et al., "Quantitative sensory testing in the German Research Network on Neuropathic Pain (DFNS): standardized protocol and reference values," Pain, vol. 123, no. 3, pp. 231-243, 2006.

[66] S. Cathcart, A. H. Winefield, P. Rolan, and K. Lushington, "Reliability of temporal summation and diffuse noxious inhibitory control," Pain Research and Management, vol. 14, no. 6, pp. 433438, 2009.

[67] H. Nie, L. Arendt-Nielsen, H. Andersen, and T. GravenNielsen, "Temporal summation of pain evoked by mechanical stimulation in deep and superficial tissue," Journal of Pain, vol. 6, no. 6, pp. 348-355, 2005.

[68] M. Granot, I. Weissman-Fogel, Y. Crispel et al., "Determinants of endogenous analgesia magnitude in a diffuse noxious inhibitory control (DNIC) paradigm: do conditioning stimulus painfulness, gender and personality variables matter?" Pain, vol. 136, no. 1-2, pp. 142-149, 2008.

[69] S. Lautenbacher, S. Roscher, and F. Strian, "Inhibitory effects do not depend on the subjective experience of pain during heterotopic noxious conditioning stimulation (HNCS): a contribution to the psychophysics of pain inhibition," The European Journal of Pain, vol. 6, no. 5, pp. 365-374, 2002.

[70] M. Valeriani, D. Le Pera, D. Restuccia et al., "Segmental inhibition of cutaneous heat sensation and of laser-evoked potentials by experimental muscle pain," Neuroscience, vol. 136, no. 1, pp. 301-309, 2005.

[71] C. M. Campbell, C. R. France, M. E. Robinson, H. L. Logan, G. R. Geffken, and R. B. Fillingim, "Ethnic differences in diffuse noxious inhibitory controls," Journal of Pain, vol. 9, no. 8, pp. 759-766, 2008.

[72] R. Staud, M. E. Robinson, C. J. Vierck, and D. D. Price, "Diffuse noxious inhibitory controls (DNIC) attenuate temporal summation of second pain in normal males but not in normal females or fibromyalgia patients," Pain, vol. 101, no. 1-2, pp. 167174, 2003.

[73] P. Svensson, C. H. Hashikawa, and K. L. Casey, "Site- and modality-specific modulation of experimental muscle pain in humans," Brain Research, vol. 851, no. 1-2, pp. 32-38, 1999.

[74] B. Tuveson, A. S. Leffler, and P. Hansson, "Time dependant differences in pain sensitivity during unilateral ischemic pain provocation in healthy volunteers," The European Journal of Pain, vol. 10, no. 3, pp. 225-232, 2006.

[75] D. D. Price and R. Dubner, "Mechanisms of first and second pain in the peripheral and central nervous systems," Journal of Investigative Dermatology, vol. 69, no. 1, pp. 167-171, 1977.

[76] R. Staud, C. E. Bovee, M. E. Robinson, and D. D. Price, "Cutaneous C-fiber pain abnormalities of fibromyalgia patients are specifically related to temporal summation," Pain, vol. 139, no. 2, pp. 315-323, 2008.

[77] P. H. Finan, L. F. Buenaver, S. C. Bounds et al., "Discordance between pain and radiographic severity in knee osteoarthritis findings from quantitative sensory testing of central sensitization," Arthritis and Rheumatism, vol. 65, no. 2, pp. 363-372, 2013.

[78] L. Arendt-Nielsen and S. Petersen-Felix, "Wind-up and neuroplasticity: is there a correlation to clinical pain?" European Journal of Anaesthesiology, Supplement, vol. 12, no. 10, pp. 1-7, 1995.

[79] M. C. Ribeiro-Dasilva, B. R. Goodin, and R. B. Fillingim, "Differences in suprathreshold heat pain responses and selfreported sleep quality between patients with temporomandibular joint disorder and healthy controls," The European Journal of Pain, vol. 16, no. 7, pp. 983-993, 2012.

[80] Y. Tobbackx, M. Meeus, L. Wauters et al., "Does acupuncture activate endogenous analgesia in chronic whiplash-associated disorders? A randomized crossover trial," The European Journal of Pain, vol. 17, no. 2, pp. 279-289, 2013.

[81] G. G. Zhang, C. Yu, W. Lee, L. Lao, K. Ren, and B. M. Berman, "Involvement of peripheral opioid mechanisms in electroacupuncture analgesia," Explore, vol. 1, no. 5, pp. 365-371, 2005.

[82] C. J. Woolf and S. W. N. Thompson, "The induction and maintenance of central sensitization is dependent on N-methyl-Daspartic acid receptor activation; implications for the treatment of post-injury pain hypersensitivity states," Pain, vol. 44, no. 3, pp. 293-299, 1991.

[83] D. Yarnitsky, "Conditioned pain modulation (the diffuse noxious inhibitory control-like effect): its relevance for acute and chronic pain states," Current Opinion in Anaesthesiology, vol. 23, no. 5, pp. 611-615, 2010.

[84] D. Yarnitsky, L. Arendt-Nielsen, and D. Bouhassira, "Recommendations on terminology and practice of psychophysical DNIC testing," The European Journal of Pain, vol. 14, no. 4, article 339, 2010.

[85] R. Moont, Y. Crispel, R. Lev, D. Pud, and D. Yarnitsky, "Temporal changes in cortical activation during distraction from pain: a comparative LORETA study with conditioned pain modulation," Brain Research, vol. 1435, pp. 105-117, 2012.

[86] R. Moont, D. Pud, E. Sprecher, G. Sharvit, and D. Yarnitsky, "Pain inhibits pain' mechanisms: is pain modulation simply due to distraction?" Pain, vol. 150, no. 1, pp. 113-120, 2010.

[87] D. le Bars and J.-C. Willer, "Pain modulation triggered by highintensity stimulation: implication for acupuncture analgesia?" International Congress Series, vol. 1238, pp. 11-29, 2002. 
[88] D. Le Bars, A. H. Dickenson, and J. M. Besson, "Diffuse noxious inhibitory controls (DNIC). I. Effects on dorsal horn convergent neurones in the rat," Pain, vol. 6, no. 3, pp. 283-304, 1979.

[89] D. Le Bars, A. H. Dickenson, and J. M. Besson, "Diffuse noxious inhibitory controls (DNIC). II. Lack of effect on nonconvergent neurones, supraspinal involvement and theoretical implications," Pain, vol. 6, no. 3, pp. 305-327, 1979.

[90] O. Gall, D. Bouhassira, D. Chitour, and D. Le Bars, "Involvement of the caudal medulla in negative feedback mechanisms triggered by spatial summation of nociceptive inputs," Journal of Neurophysiology, vol. 79, no. 1, pp. 304-311, 1998.

[91] L. Villanueva, M. Peschanski, B. Calvino, and D. Le Bars, "Ascending pathways in the spinal cord involved in triggering diffuse noxious inhibitory controls in the rat," Journal of Neurophysiology, vol. 55, no. 1, pp. 34-55, 1986.

[92] L. Villanueva, D. Chitour, and D. Le Bars, "Involvement of the dorsolateral funiculus in the descending spinal projections responsible for diffuse noxious inhibitory controls in the rat," Journal of Neurophysiology, vol. 56, no. 4, pp. 1185-1195, 1986.

[93] D. Le Bars, L. Villanueva, D. Bouhassira, and J. C. Willer, "Diffuse noxious inhibitory controls (DNIC) in animals and in man," Patologicheskaya Fiziologiya i Eksperimentalnaya Terapiya, no. 4, pp. 55-65, 1992.

[94] A. Roby-Brami, B. Bussel, J. C. Willer, and D. LeBars, "An electrophysiological investigation into the pain-relieving effects of heterotopic nociceptive stimuli. Probable involvement of a supraspinal loop," Brain, vol. 110, no. 6, pp. 1497-1508, 1987.

[95] J. C. Willer, A. Roby, and D. Le Bars, "Psychophysical and electrophysiological approaches to the pain-relieving effects of heterotopic nociceptive stimuli," Brain, vol. 107, no. 4, pp. 10951112,1984

[96] S. Lautenbacher and G. B. Rollman, "Possible deficiencies of pain modulation in fibromyalgia," Clinical Journal of Pain, vol. 13, no. 3, pp. 189-196, 1997.

[97] A. Pielsticker, G. Haag, M. Zaudig, and S. Lautenbacher, "Impairment of pain inhibition in chronic tension-type headache," Pain, vol. 118, no. 1-2, pp. 215-223, 2005.

[98] C. H. Wilder-Smith, D. Schindler, K. Lovblad, S. M. Redmond, and A. Nirkko, "Brain functional magnetic resonance imaging of rectal pain and activation of endogenous inhibitory mechanisms in irritable bowel syndrome patient subgroups and healthy controls," Gut, vol. 53, no. 11, pp. 1595-1601, 2004.

[99] E. Kosek and G. Ordeberg, "Lack of pressure pain modulation by heterotopic noxious conditioning stimulation in patients with painful osteoarthritis before, but not following, surgical pain relief," Pain, vol. 88, no. 1, pp. 69-78, 2000.

[100] R. R. Nir, Y. Granovsky, D. Yarnitsky, E. Sprecher, and M. Granot, "A psychophysical study of endogenous analgesia: the role of the conditioning pain in the induction and magnitude of conditioned pain modulation," The European Journal of Pain, vol. 15, no. 5, pp. 491-497, 2011.

[101] K. Fujii, K. Motohashi, and M. Umino, "Heterotopic ischemic pain attenuates somatosensory evoked potentials induced by electrical tooth stimulation: diffuse noxious inhibitory controls in the trigeminal nerve territory," The European Journal of Pain, vol. 10, no. 6, pp. 495-504, 2006.

[102] J. C. Willer, T. De Broucker, and D. Le Bars, "Encoding of nociceptive thermal stimuli by diffuse noxious inhibitory controls in humans," Journal of Neurophysiology, vol. 62, no. 5, pp. 1028-1038, 1989.

[103] Z. Bing, L. Villanueva, and D. Le Bars, "Acupuncture and diffuse noxious inhibitory controls: naloxone-reversible depression of activities of trigeminal convergent neurons," Neuroscience, vol. 37, no. 3, pp. 809-818, 1990.

[104] J. Schliessbach, E. van der Klift, A. Siegenthaler, L. ArendtNielsen, M. Curatolo, and K. Streitberger, "Does acupuncture needling induce analgesic effects comparable to diffuse noxious inhibitory controls?" Evidence-Based Complementary and Alternative Medicine, vol. 2012, Article ID 785613, 2012.

[105] K. Streitberger and J. Kleinhenz, "Introducing a placebo needle into acupuncture research," The Lancet, vol. 352, no. 9125, pp. 364-365, 1998.

[106] K. K. S. Hui, E. E. Nixon, M. G. Vangel et al., "Characterization of the "deqi" response in acupuncture," BMC Complementary and Alternative Medicine, vol. 7, article 33, 2007.

[107] J. M. Helms, Acupuncture Energetics, Medical Acupuncture Publishers, 1995.

[108] C. D. King, F. Wong, T. Currie, A. P. Mauderli, R. B. Fillingim, and J. L. Riley, "Deficiency in endogenous modulation of prolonged heat pain in patients with irritable bowel syndrome and temporomandibular disorder," Pain, vol. 143, no. 3, pp. 172178, 2009.

[109] R. Staud, "Abnormal endogenous pain modulation is a shared characteristic of many chronic pain conditions," Expert Review of Neurotherapeutics, vol. 12, no. 5, pp. 577-585, 2012.

[110] M. Curatolo, L. Arendt-Nielsen, and S. Petersen-Felix, "Evidence, mechanisms, and clinical implications of central hypersensitivity in chronic pain after whiplash injury," Clinical Journal of Pain, vol. 20, no. 6, pp. 469-476, 2004.

[111] J. D. Greenspan, G. D. Slade, E. Bair et al., "Pain sensitivity risk factors for chronic TMD: descriptive data and empirically identified domains from the OPPERA case control study," Journal of Pain, vol. 12, no. 11 Supplement, pp. T61-T74, 2011.

[112] E. Eisenberg, A. Midbari, M. Haddad, and D. Pud, "Predicting the analgesic effect to oxycodone by "static" and "dynamic" quantitative sensory testing in healthy subjects," Pain, vol. 151, no. 1, pp. 104-109, 2010.

[113] R. Landau, J. C. Kraft, L. Y. Flint et al., "An experimental paradigm for the prediction of Post-Operative Pain (PPOP)," Journal of Visualized Experiments, no. 35, 2010.

[114] C. Valencia, R. B. Fillingim, and S. Z. George, "Suprathreshold heat pain response is associated with clinical pain intensity for patients with shoulder pain," Journal of Pain, vol. 12, no. 1, pp. 133-140, 2011.

[115] D. Yarnitsky, Y. Crispel, E. Eisenberg et al., "Prediction of chronic post-operative pain: pre-operative DNIC testing identifies patients at risk," Pain, vol. 138, no. 1, pp. 22-28, 2008.

[116] D. Yarnitsky, M. Granot, H. Nahman-Averbuch, M. Khamaisi, and Y. Granovsky, "Conditioned pain modulation predicts duloxetine efficacy in painful diabetic neuropathy," Pain, vol. 153, no. 6, pp. 1193-1198, 2012.

[117] L. Arendt-Nielsen, J. B. Frøkjær, C. Staahl et al., “ Effects of gabapentin on experimental somatic pain and temporal summation," Regional Anesthesia and Pain Medicine, vol. 32, no. 5, pp. 382-388, 2007.

[118] D. Kleinböhl, R. Görtelmeyer, H. J. Bender, and R. Hölzl, "Amantadine sulfate reduces experimental sensitization and pain in chronic back pain patients," Anesthesia and Analgesia, vol. 102, no. 3, pp. 840-847, 2006.

[119] D. Pud, E. Eisenberg, A. Spitzer, R. Adler, G. Fried, and D. Yarnitsky, "The NMDA receptor antagonist amantadine reduces surgical neuropathic pain in cancer patients: a double blind, randomized, placebo controlled trial," Pain, vol. 75, no. 2-3, pp. 349-354, 1998. 
[120] C. Valencia, L. L. Kindler, R. B. Fillingim, and S. Z. George, "Investigation of central pain processing in shoulder pain: converging results from 2 musculoskeletal pain models," Journal of Pain, vol. 13, no. 1, pp. 81-89, 2012.

[121] C. J. Vierck, R. Staud, D. D. Price, R. L. Cannon, A. P. Mauderli, and A. D. Martin, "The Effect of maximal exercise on temporal summation of second pain (windup)in patients with fibromyalgia syndrome," Journal of Pain, vol. 2, no. 6, pp. 334-344, 2001.

[122] S. Iyengar, A. A. Webster, S. K. Hemrick-Luecke, J. Y. Xu, and R. M. A. Simmons, "Efficacy of duloxetine, a potent and balanced serotonin-norepinephrine reuptake inhibitor in persistent pain models in rats," Journal of Pharmacology and Experimental Therapeutics, vol. 311, no. 2, pp. 576-584, 2004.

[123] L. Arendt-Nielsen, H. Mansikka, C. Staahl et al., "A translational study of the effects of ketamine and pregabalin on temporal summation of experimental pain," Regional Anesthesia and Pain Medicine, vol. 36, no. 6, pp. 585-891, 2011.

[124] M. J. Alappattu, M. D. Bishop, J. E. Bialosky, S. Z. George, and M. E. Robinson, "Stability of behavioral estimates of activitydependent modulation of pain," Journal of Pain Research, vol. 4, pp. 151-157, 2011.

[125] M. Granot, Y. Granovsky, E. Sprecher, R. R. Nir, and D. Yarnitsky, "Contact heat-evoked temporal summation: tonic versus repetitive-phasic stimulation," Pain, vol. 122, no. 3, pp. 295-305, 2006.

[126] D. D. Price, J. Mao, H. Frenk, and D. J. Mayer, "The N-methyl$\mathrm{D}$-aspartate receptor antagonist dextromethorphan selectively reduces temporal summation of second pain in man," Pain, vol. 59, no. 2, pp. 165-174, 1994.

[127] C. Maier, R. Baron, T. R. Tölle et al., "Quantitative sensory testing in the German Research Network on Neuropathic Pain (DFNS): somatosensory abnormalities in 1236 patients with different neuropathic pain syndromes," Pain, vol. 150, no. 3, pp. 439-450, 2010. 


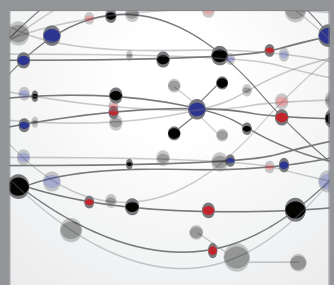

The Scientific World Journal
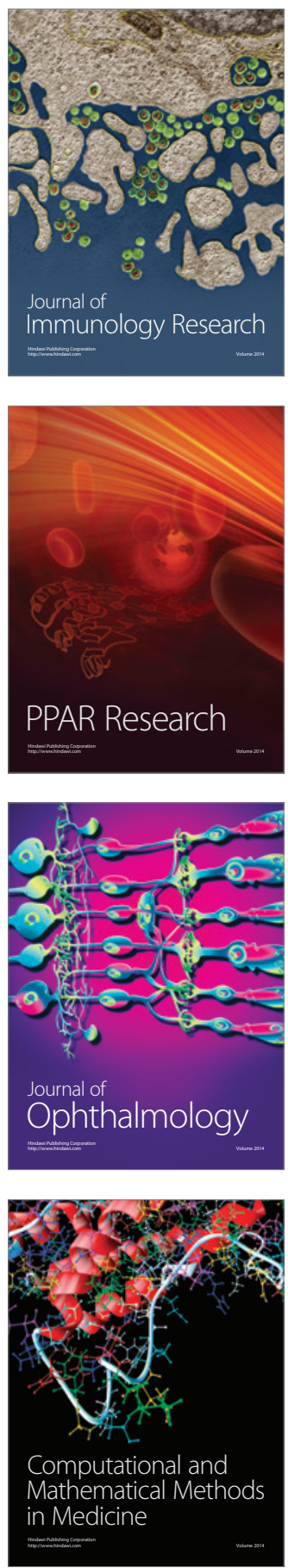

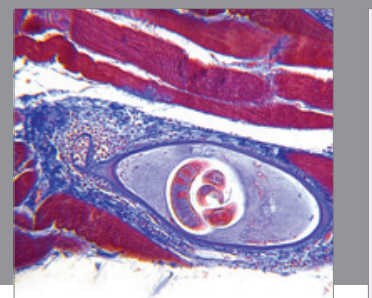

Gastroenterology

Research and Practice
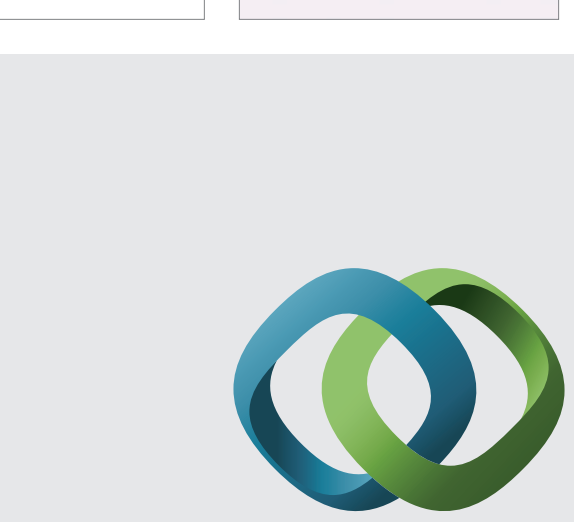

\section{Hindawi}

Submit your manuscripts at

http://www.hindawi.com
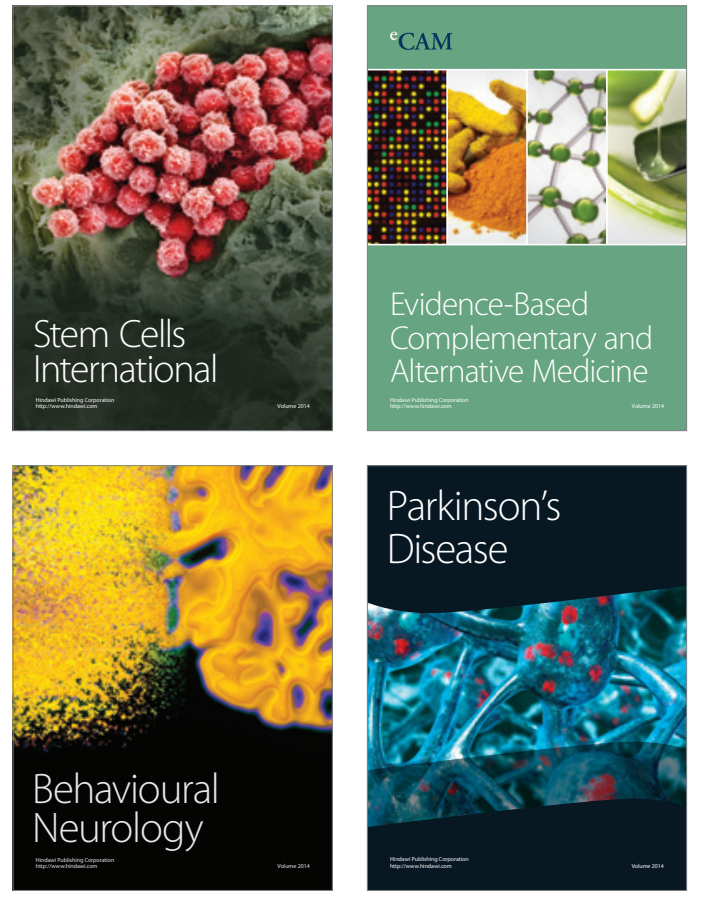
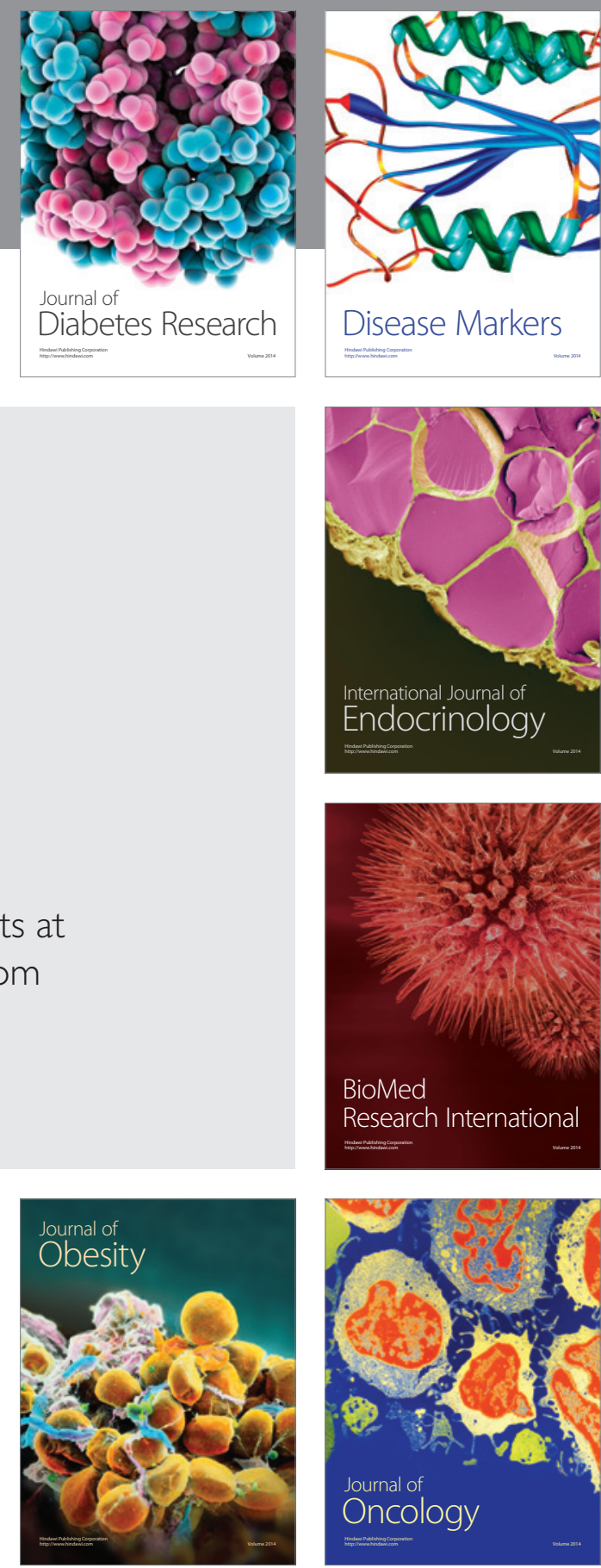

Disease Markers
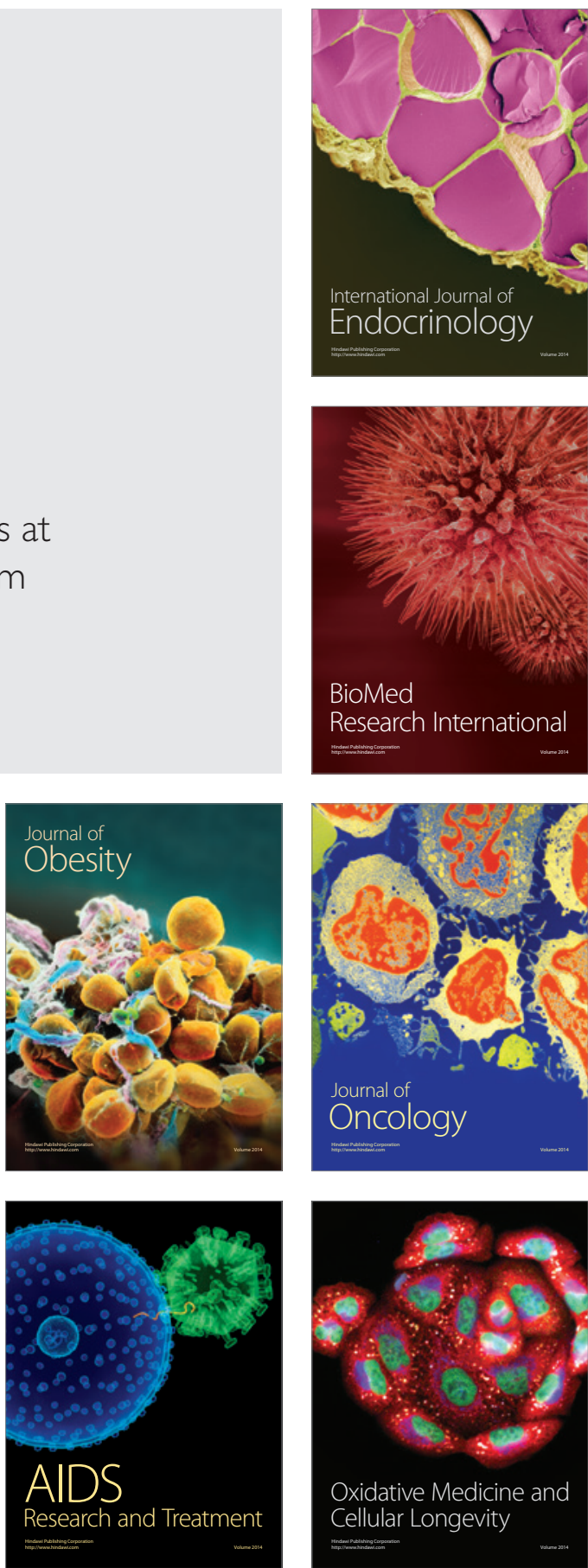